EPiC Series in Engineering
Volume 3, 2018, Pages 1718-1726
HIC 2018. 13th International
Conference on Hydroinformatics

\title{
Relationship between extreme rainfall and surface temperature in Sicily (Italy)
}

\author{
Dario Pumo $^{1 *}$, Giuseppina Carlino ${ }^{1}$, Elisa Arnone ${ }^{2}$ and Leonardo V. Noto ${ }^{1}$ \\ ${ }^{1}$ Dipartimento di Ingegneria Civile, Ambientale, Aerospaziale, dei Materiali, Università degli \\ Studi di Palermo, Viale delle Scienze, Edificio 8, 90128, Palermo, Italy \\ ${ }^{2}$ AMIGO s.r.l., Via Flaminia 48, I-00196, Roma, Italy \\ dario.pumo@unipa.it, giuseppina.carlino@community.unipa.it, \\ elisa.arnonedamigoclimate.com, leonardo.noto@unipa.it
}

\begin{abstract}
The study of the relationship between extreme rainfall events and surface temperature represents an important issue in hydrology and meteorology and it could be of capital importance for evaluating the effect of global warming on future precipitation. Various approaches have been tested across different parts of the world, and, in many cases, it has been observed an intensification of precipitation with increasing temperature consistently with the thermodynamic Clausius-Clapeyron relation (CC-rate of $6-7 \%{ }^{\circ} \mathrm{C}^{-1}$ ), according to which a warmer atmosphere is capable of holding more moisture. Nevertheless, in different locations, the scaling rate between temperature and extreme precipitation has resulted significantly different with respect to the CC-rate, in some cases sensibly higher (super-CC) and in other relevantly lower (sub-CC). In this work, an analysis of the scaling relationship between sub-daily extreme rainfall events and surface temperature is carried out, using data from a large number of rain and temperature gauges across Sicily (Italy). Results highlight the relevant importance of some modeling choices and, particularly, of rainfall duration, for this type of analysis in semi-arid region. An overall sub-CC scaling rate has been detected for most part of the region.
\end{abstract}

\section{Introduction}

One of the most significant implications of a warming climate in many parts of the world is an intensification of extreme precipitation. More frequent short-duration extreme rainfall events can lead

\footnotetext{
${ }^{*}$ First and corresponding author
} 
to numerous hazards, from flooding to landslides, with growing risks for human life and damage to buildings and infrastructures.

Atmospheric temperature strongly influences rainfall intensity, since warmer air has the potential to provide more moisture to the rainfall process. The intensity of rainfall events is, in fact, strictly correlated to the amount of vapor held in atmosphere and the physical conditions of the air. The theoretical basis of the relationship that links air temperature and atmospheric humidity is provided by the Clausius-Clapeyron relation (CC), according to which, if relative humidity remains constant, then atmospheric moisture will increase at a rate that follows the saturation vapor pressure dependency on temperature (i.e., $6-7 \%{ }^{\circ} \mathrm{C}^{-1}$ ). Under the assumption that during extreme events, all water vapor in the air (or a relevant and constant fraction of it) is converted into rain, then precipitation for extreme events should scale with the CC rate.

Interestingly, various observational studies in different parts of the world have exhibited scaling rate not constant with land surface temperature, different from the theoretical $\mathrm{CC}$ rate and with values both higher (super-CC) and lower (sub-CC).

One of earlier works analyzing the scaling relationship between precipitation and temperature, using data from the Netherlands, tried to explain how the extreme percentiles of hourly rainfall vary depending on surface temperature (Lenderink \& Van Meijgaard, 2008). The main outcome was that the $99^{\text {th }}$ and higher percentiles increased with temperature at approximately the CC rate for temperatures up to $12^{\circ} \mathrm{C}$ and at double rate for temperatures up to $22^{\circ} \mathrm{C}$. A similar methodology was subsequently used to analyze the scaling rate in various regions across the world, from Europe (Berg, Moseley, \& Haerter, 2013; Berg \& Haerter, 2013; Blenkinsop, Chan, Kendon, Roberts, \& Folwer, 2015; Loriaux, Lenderink, De Roode, \& Siebesma, 2013) to Australia (Hardwick Jones, Westra, \& Sharma, 2010), from Nord America (Shaw, Royem, \& Riha, 2011; Mishra, Wallace, \& Lettenmaier, 2012) to Asia (Yu \& Li, 2012; Utsumi, Seto, Kanae, Maeda, \& Oki, 2011). Nevertheless, there are very few evidences of this type of analysis for arid or semi-arid areas, where scaling frequently appears to be lower than the CC rate (Pall, Allen, \& Stone, 2007; Wentz, Ricciardulli, Hilburn, \& Mears, 2007; O'Gorman \& Muller, 2010).

Recent studies (Blenkinsop, Chan, Kendon, Roberts, \& Folwer, 2015; Hardwick Jones, Westra, \& Sharma, 2010) have shown that the relationship between mean daily temperature and the $99^{\text {th }}$ percentile of maximum hourly precipitation cannot always be fully interpreted by a linear regression model. Sometimes LOESS (LOcally-wEighted Scatter-plot Smoothing) approach (Cleveland, 1979) estimated relationship has been also used to describe the scaling behavior (Blenkinsop, Chan, Kendon, Roberts, \& Folwer, 2015). Some studies investigated the role of various modeling choices; for instance, the scaling rate may vary widely with the selected percentile and with data time resolution. In particular, scaling rate has been found to be approximately constant for sub-hourly durations and then reduces with longer durations, and, furthermore, it increases with increasing percentile (Hardwick Jones, Westra, \& Sharma, 2010).

Also seasonality has an important role in the scaling relationship for daily precipitation; a seasonal variability in the temperature dependence of precipitation intensity has been observed, for instance, in (Sherwood, Roca, Weckwerth, \& Andronova, 2010), where a general increase in winter and a decrease in summer have been found (Westra, et al., 2014; Panthou, Mailhot, Laurence, \& Talbot, 2014).

In this study, we refer to the most consolidated and used modelling framework (Berg, Moseley, \& Haerter, 2013; Berg \& Haerter, 2013; Loriaux, Lenderink, De Roode, \& Siebesma, 2013; Blenkinsop, Chan, Kendon, Roberts, \& Folwer, 2015; Hardwick Jones, Westra, \& Sharma, 2010; Molnar, Fatichi, Gaal, Szolgay, \& Burlando, 2015), using a similar approach to investigate the scaling relationship between sub-daily extreme rainfall events and surface temperature in Sicily, a semi-arid region of southern Italy. The role of different factors, such as the duration of rainfall maximum depth, the type of regression models and the climate seasonality are also analyzed. 


\section{Data and Methods}

The area study is the Sicily region (southern Italy), where a discrete spatial variability in the elevation (Figure 1a) concurs into generating a rather significative spatial variability also in some climate variables such as precipitation (Figure 1b) and temperature (Figure 1c).

The dataset is constituted by data collected by the regional agency SIAS (Servizio Informativo Agrometeorologico Siciliano; i.e. Agro-meteorological Information Service of Sicily) from 2003 to 2015 at 107 temperature and rain gauge stations spread over the entire region (Errore. L'origine riferimento non è stata trovata.d). The original data time-resolution was hourly for temperature (for a total sample size of 12,192,864 data) and 10 minutes for rainfall (for a total sample size of $73,157,184$ data). A data pre-processing procedure has been preliminarily applied to each gauge station records in order to identify missing or suspicious data (individual year with more than $15 \%$ missing data have been rejected). Stations with less than 10 years of simultaneous temperatureprecipitation data have been excluded; at the end of this procedure, the resulting dataset included data from 93 gauges.

The adopted methodology can be summarized through the following steps:

- identification, for each gauge station, of all the rainy days (i.e. days with not null rainfall), and, for each of them, computation of the mean daily temperature and the maximum rainfall for the duration of 10,30 and 60 minutes (hereafter referred to as $\mathrm{P}_{10}$, $\mathrm{P}_{30}$ and $\left.\mathrm{P}_{60}\right)$;

- constitution, for each gauge station and rainfall duration, of three seasonal subsamples with couples of corresponding (i.e. both referred to the same day) values of mean temperature and rainfall maximum: 1) Unique Season sample (data from the entire hydrological year); 2) Dry Season sample (data from April to September); 3) Wet Season sample (data of the remaining part of the year);

- distinction between single gauge analysis and pooled gauges analysis: for this last case, all the different seasonal subsamples from the various stations have been pooled together and studied as an unique regional seasonal sample;

- $\quad$ at the level of both single gauge and pooled gauges analysis, each sample has been first subdivided in a number (i.e., 10) of temperature bins, using an equal number (i.e. variable bin widths) classification (Blenkinsop, Chan, Kendon, Roberts, \& Folwer, 2015). A couple of values have been then associated to each bin (i.e., 10 couples for each sample): the $99^{\text {th }}$ percentile of rainfall maxima (q99) and the median temperature (T) for each bin;

- regression analysis to determine the fitting curve of the q99-T data derived at the previous step for each sample and estimate the corresponding rates; different regression models have been considered such as the exponential regression, the Two-Segment Piecewise Regression and the LOESS regression (Cleveland, 1979).

A further analysis has been carried out to investigate the role of the considered percentile and number of bins, comparing scaling rates arising from: (i) a fixed number of bins (i.e. 10) and multiple quantiles (q50, q75, q90 and q99); and (ii) a fixed quantile (i.e. q99) and a different number of bins (from 8 to 12). It is important to point out that only regression models (least-squared linear regression using the $\log$ of precipitation) with coefficient of determination $\left(\mathrm{R}^{2}\right)$ higher than a fixed threshold (assumed equal to 0.55) are considered as sufficiently adequate to represent the searched relationships; thus, scaling rates of models having lower $\mathrm{R}^{2}$ are neglected from successive analyses and statistics computation. 

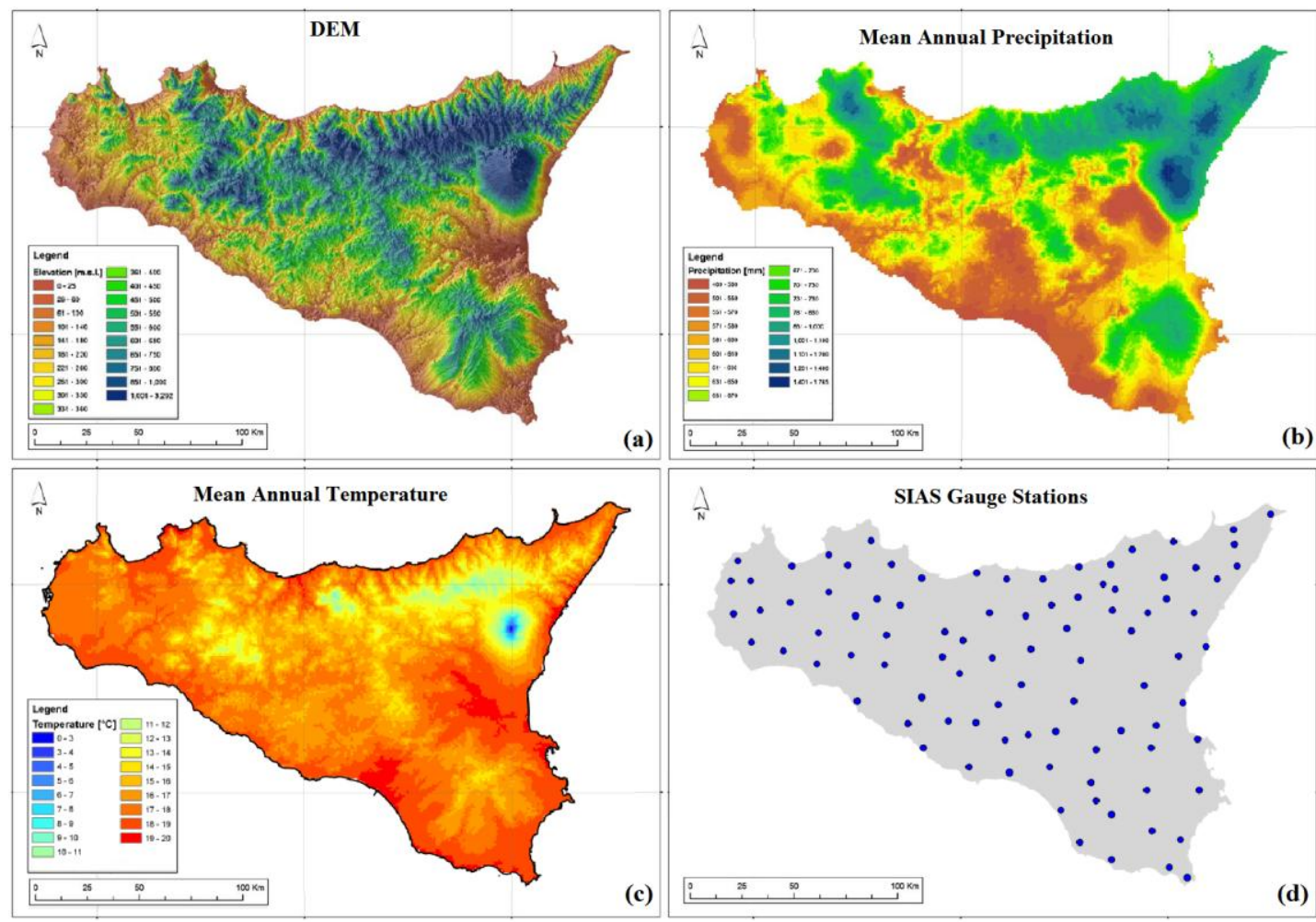

(b)

Figure 1: DEM (a); Mean Annual Precipitation (b) and Mean Annual Temperature (c) Regional map of SIAS weather stations (d).

\section{Results}

\subsection{Single Gauges Analysis}

A first analysis has investigated the capability of regression models to interpret the relationship between rainfall percentiles (q99) and median temperature (T) for each bin (considered number of bins $=10$ ). From the single gauge analysis, one can observe a different percentage of stations where the adoption of exponential regression models is suitable, depending on the considered season (Unique, Dry or Wet Season) and rainfall duration (10, 3060 minutes).

As it can be noticed from Figure 2 the percentage of considered stations (i.e., stations with associated a model with $\mathrm{R}^{2}>0.55$ ) is higher for the Unique and Wet Season, probably due to a higher samples size related to the higher frequency of rainy days in Sicily during the autumn and winter months. Rainy days are usually significantly reduced in spring and are almost absent during the summer and this implies, for many gauges in Sicily, a not sufficient consistence of the dataset during the Dry Season that, in turn, could affect the $\mathrm{R}^{2}$ of regression models. This would explain the reduction of the percentage of considered stations (about -60\%) with respect to the Unique Season, and it could be a very important aspect to be considered for analyses in arid regions. Figure 2 shows that the percentage of considered stations increases with decreasing rainfall durations. This might be related to an overall stronger relationships between extreme precipitation and surface temperature when shorter events are considered; for several stations the regression models applied to $\mathrm{P}_{60}$ have had 
slightly unsatisfying $R^{2}(\leq 0.55)$, while passing from $P_{60}$ to the $P_{10}$ for the same stations (i.e. same original dataset), the corresponding regression model has provided acceptable $\mathrm{R}^{2}(>0.55)$.

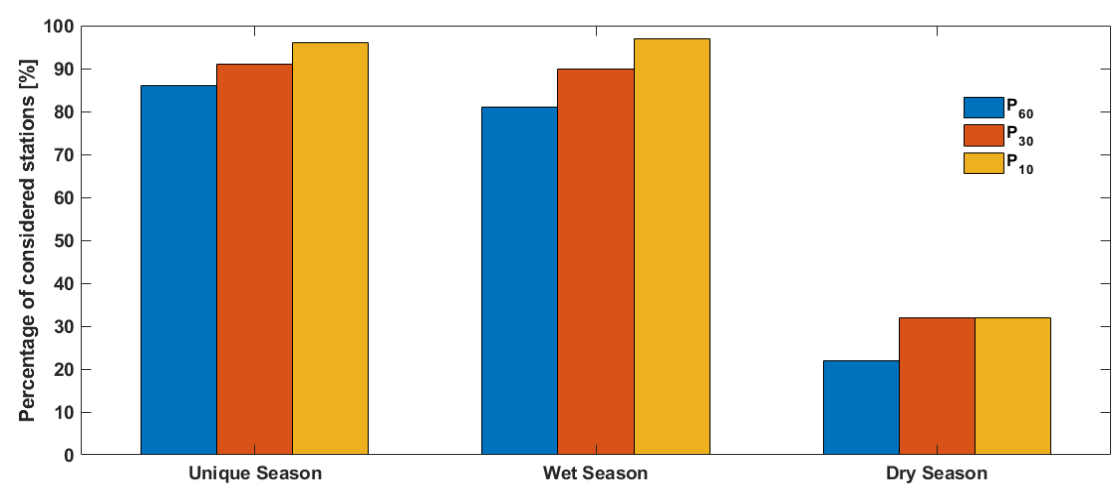

Figure 2: Percentage of considered stations $\left(\mathrm{R}^{2}>0.55\right)$ for the different subsamples (Unique Season, Wet Season, Dry Season) and rainfall durations (10, 30, 60 minutes). The total number of stations analyzed is 93.

Figure 3a shows an example of exponential regression (linear regression in a semi-log plot) for the Unique Season sample of $\mathrm{P}_{30}$ recorded at the station ID 750 (Palermo Uditore). In this case, a typical sub-CC rate behaviour ( $\mathrm{rate}=3,7 \%{ }^{\circ} \mathrm{C}^{-1}$ ) occurs.

In Figure 3b, the average scaling rate over all the stations for different considered percentiles (q50, q75, q90 and q99) are plotted. The figure refers to the Wet Season and $P_{10}$ and shows how the scalingrate considerably increases with increasing percentile. The same behavior has been also found for the other seasons and for all the rainfall durations (not shown in figure) and is consistent with what found by (Hardwick Jones, Westra, \& Sharma, 2010).

Figure $3 \mathrm{c}$ shows the dependence of the scaling behavior on the number of bins; it displays the average scaling rate over all the stations derived considering a different number of bins (from 8 to 12) for the case of Unique Season and $\mathrm{P}_{10}$. The analysis showed that, for the Unique Season (as well as for the Wet Season) the scaling rate slightly decreases when the considered number of bins increases; an opposite behavior has been found for the Dry Season (not shown in figure), probably due to the lower samples size that could affect the analysis when temperature domain is subdivided into more bins.

The analyses discussed hereafter have been performed considering q99 and a total number of bins equal to 10, according to the modeling framework adopted in (Blenkinsop, Chan, Kendon, Roberts, \& Folwer, 2015).
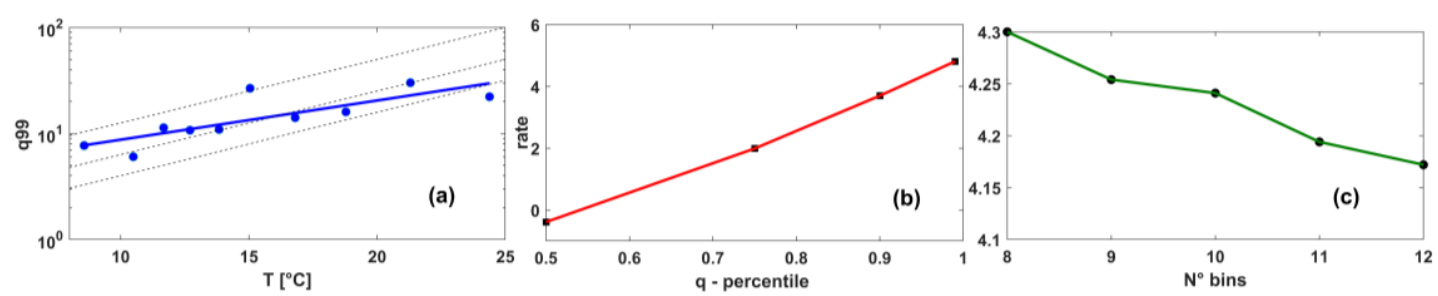

Figure 3: (a) Scaling relationships for the station ID 750: sample for the Unique Season and $\mathrm{P}_{30}$, black dashed lines refer to the CC-rate, while blue line refers to the least squared fitted linear regression model on the $\log$ of precipitation. Average scaling rate over all the stations as a function of (b) the percentiles (q50, q75, q90, q99, Wet Season, $\mathrm{P}_{10}, 10$ bins) and (c) the number of bins (q99, $\mathrm{N}^{\circ}$ bins from 8 to 12, Unique Season, $\mathrm{P}_{10}$ ). 
In Figure 4, the empirical cumulative distribution functions (ecdfs) of the scaling rates found through the exponential regression (with $\mathrm{R}^{2}>0.55$ ) across all the gauges of the region are represented. Different colors refer to the different seasons, upper plot refers to $\mathrm{P}_{60}$, while middle and bottom plots refer to $\mathrm{P}_{30}$ and $\mathrm{P}_{10}$, respectively. From the Figure 4, a high variability of the scaling across the Sicily can be noticed for all the considered seasons and durations, with rates positive and ranging from 2 $\%{ }^{\circ} \mathrm{C}^{-1}$ to $8 \%{ }^{\circ} \mathrm{C}^{-1}$.

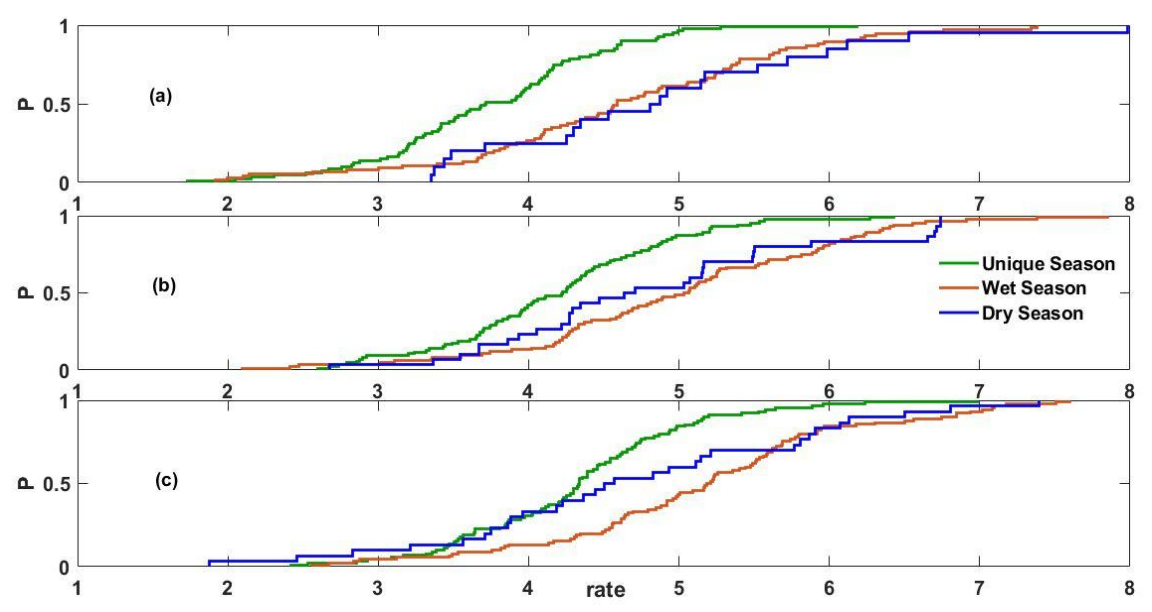

Figure 4: Ecdfs of the scaling rates of all the Sicilian gauges for the three considered rainfall durations: (a) $\mathrm{P}_{60}$; (b) $\mathrm{P}_{30}$; (c) $\mathrm{P}_{10}$. Different colors refer to the three seasons: Unique, Wet and Dry Season.

For all the analyzed durations, the scaling rate for the Wet and Dry Season resulted, on average, higher than for the Unique Season; this fact is strongly related to the much higher temperature variability characterizing the Unique Season that implies a smoothing of the scaling rate, and highlights the importance of perform seasonal analyses for regions characterized by high seasonal variability in temperature. An important aspect is also the different types of rainfall events characterizing the different seasons; in particular, during the warmer season in Sicily (especially during the summer) there is a prevalence of convective rainfall, which is more sensitive to temperature variations with respect to the large-scale stratiform precipitation that usually characterizes the colder months. This could partially explain the increase of rates found for higher temperatures, especially during the Dry Season.

The scaling rate has resulted also particularly sensitive to the rainfall event duration; in particular, in accordance with (Hardwick Jones, Westra, \& Sharma, 2010), it has been found to significantly increase with shorter precipitation durations (i.e. from $\mathrm{P}_{60}$ to $\mathrm{P}_{10}$ ) for the Unique and Wet Season, while the scaling for Dry Season has resulted almost insensitive to rainfall durations with only slightly lower scaling rates for 10 minutes events

\subsection{Two-Segment Piecewise Regression analysis}

The Two-Segment Piecewise Regression analysis has been performed at the level of single gauge by using an automatic code implemented in Matlab (MathWorks) and capable to: (i) detect the breakpoint in the cloud of data points, dividing the temperature range into two parts; (ii) fit a least-squares linear regression model to each; (iii) derive the two scaling rates, specific for each segment. In Figure 5, two explicative cases, referred to the Wet Season and $\mathrm{P}_{10}$, are represented, comparing the scaling rates obtained for the segments on the left $\left(\alpha_{1}\right)$ and on the right $\left(\alpha_{2}\right)$ of the break-point (see dashed red 
lines), with that resulting from the Exponential Regression ( $\alpha$, see the solid blue lines). In particular, Figure 5a shows the results for the station ID 764, where the analysis has indicated a super-CC (i.e., $10.7 \%{ }^{\circ} \mathrm{C}^{-1}$ ) up to $12.4^{\circ} \mathrm{C}$ and a sub-CC above this temperature. A different behavior is observed for the case of Figure 5b, which shows one of the cases (ID 763) where a gauge sample cannot be adequately fitted by an Exponential Regression model $\left(R^{2}=0.25\right)$, whereas it can be successfully fitted using the Two-Segment Piecewise Regression $\left(\mathrm{R}^{2}=0.86\right.$ for the left segment and $\mathrm{R}^{2}=0.99$ for the right segment). This case is also a typical example of peak-like structure in the relationships extreme rainfall-temperature, prevalently occurring for the Dry Season, in which, above the temperature break-point (at $14.5^{\circ} \mathrm{C}$ for the case in figure) the scaling becomes negative.

a) ID 764

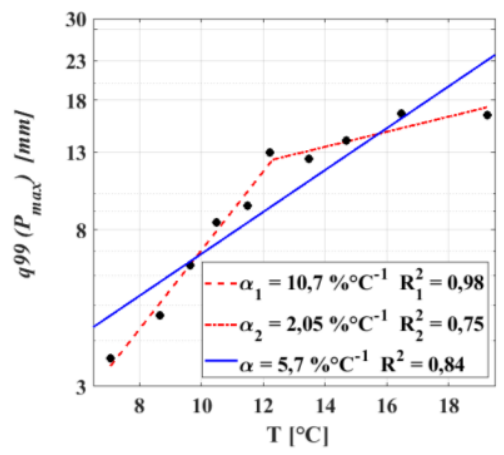

b) ID 763

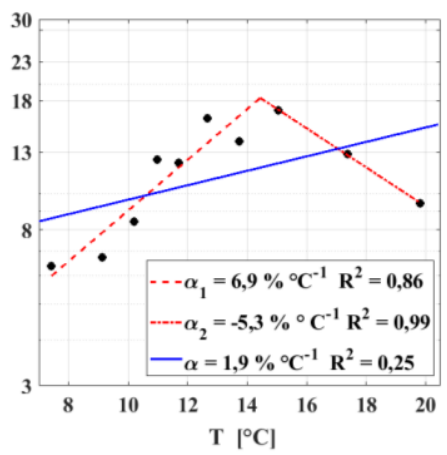

Figure 5: Red dashed lines indicate the Two-Segment Piecewise Regression for the stations ID 764 (a) and ID 763 (b), with scaling rates for each segment $\left(\alpha_{1}\right.$ and $\left.\alpha_{2}\right)$ for the case of Wet Season and $\mathrm{P}_{10}$. The solid blue lines denote the fitted Exponential Regression for each case.

\subsection{Pooled Gauges Analysis}

For the pooled gauges analysis, Sicily is considered as a single homogeneous zone and all the data recorded at all the stations are pooled into a unique sample, according to an approach also followed in (Hardwick Jones, Westra, \& Sharma, 2010; Utsumi, Seto, Kanae, Maeda, \& Oki, 2011); this obviously offers the possibility to consider a much wider sample size. Results of this analysis are visualized using a semi-log scatter density plots, fitting the nonparametric LOESS relationships, which allow to release the linearity assumptions.
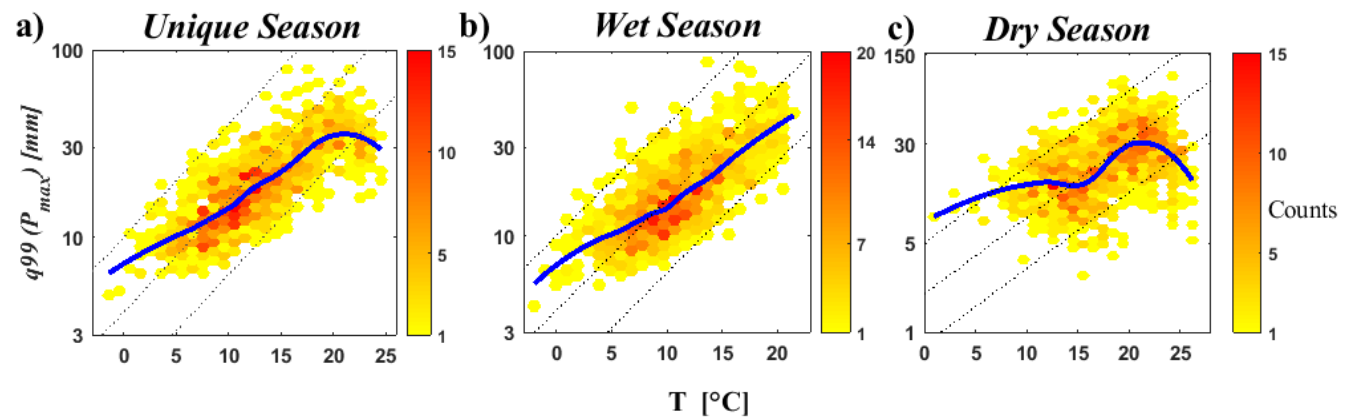

Figure 6: Density plots for pooled gauges. Solid blue lines indicate the LOESS-estimated relationship between $99^{\text {th }}$ percentile and $\mathrm{T}$, for $\mathrm{P}_{60}$ and the: (a) Unique Season; (b) Wet Season; (c) Dry Season. 
The pooled gauges analysis has confirmed the results found through the single gauge analysis. The results for hourly duration are synthesized in Figure 6, where the density plots for the Unique (Figure 6a), the Wet (Figure 6b) and the Dry Season (Figure 6c) and the estimated LOESS curves are shown. The figure exhibits an evident functional relationship between extreme rainfall events and surface temperature, almost linear (in semi-log plot) for the Wet Season, and much more elaborate for the Dry Season.

From Figure 6 it emerges that the relationship between extreme hourly precipitation and temperature varies with temperature. For all the seasons the scaling appears not to be adequately described by a linear relationship, with an evident break-point at about $10^{\circ} \mathrm{C}$ for the Wet Season and even two break-points the Unique and for the Dry Season; a negative scaling rate has been in fact found for temperature values above $22^{\circ} \mathrm{C}$.

\section{Conclusion}

This paper has examined the scaling relationship between mean daily temperature and extreme hourly and sub-hourly precipitation in Sicily, using a large and updated high-resolution dataset.

The single gauge analysis has demonstrated how rainfall duration and, to a greater extent, seasonality play a crucial role in determining the scaling rate. For the Unique and Wet Season, higher rates have been observed when shorter rainfall durations are considered, while this effect is smoothed during the Dry Season, probably due to a lower occurrence of rainfall events, which are prevalently of convective type. Significantly higher scaling rates have been obtained when the analysis is conducted at the level of single season (Wet and Dry Season) with respect to the entire hydrological year (Unique Season), demonstrating the importance, in regions characterized by a high seasonality in climate (especially in temperature), of distinguishing the different seasons.

Nevertheless, for all the seasons (including the Unique Season) and durations (from 10 minutes to 1 hours), the majority of the gauges have shown rates below the theoretical CC-rate, in accordance with the few studies present in literature for arid and semi-arid regions (Pall, Allen, \& Stone, 2007; Wentz, Ricciardulli, Hilburn, \& Mears, 2007; O'Gorman \& Muller, 2010; Sherwood, Roca, Weckwerth, \& Andronova, 2010).

This study has also highlighted the importance of using appropriate modeling assumptions in the estimation of the scaling rate. The scaling rate appears to be influenced by the number of bins in which the temperature domain is subdivided to derive the extreme rainfall values (percentiles for each bin). Nevertheless, a higher weight can be addressed to the choice of the percentile to define extreme rainfall.

The adoption of alternative approaches, such as the Piecewise Regression or the pooled gauges LOESS based - approach, has allowed to better characterize the elaborate temperature dependence of extreme rainfall in Sicily, finding various scaling rates for different sub-domains of temperature, and identifying often a peak-like behavior, not detected by the simple application of the Exponential Regression, that could be addressed to the atmospheric moisture supply limitations for the highest temperatures of the Dry Season. Differently from what observed with the exponential regression, the Two-Segment Piecewise Regression analysis has been also capable to detect super CC rates for Unique Season samples with regard to limited temperature range.

\section{References}

Berg, P., \& Haerter, J. O. (2013). Unexpected increase in precipitation intensity with temperature A result of mixing of precipitation types? Atmos. Res. , 119, 56-61. 
Berg, P., Moseley, C., \& Haerter, J. O. (2013). Strong increase in convective precipitation in response to higher temperatures. Nat. Geosci, 6, 181-5.

Blenkinsop, S., Chan, S. C., Kendon, E. K., Roberts, N. M., \& Folwer, H. J. (2015). Temperature influences on intense UK hourly precipitation and dependency on large-scale circulation. Enviromental Research Letters , 10, 054021.

Cleveland, W. S. (1979). Robust locally weighted regression and smoothing scatterplots. J. Am. Stat. Assoc. , 74 (368), 829-836.

Hardwick Jones, R., Westra, S., \& Sharma, A. (2010). Observed relationships between extreme sub-daily precipitation, surface temperature, and relative humidity. Geophys. Res. Lett. , 37, L22805.

Lenderink, G., \& Van Meijgaard, E. (2008). Increase in hourly precipitation extremes beyond expectations from temperature changes. Nat. Geosci, 1, 511-4.

Loriaux, J. M., Lenderink, G., De Roode, S. R., \& Siebesma, A. P. (2013). Understanding convective extreme precipitation scaling using observations and an entraining plume model. J. Atmos. Sci. , 70, 3641-3655.

Mishra, V., Wallace, J. M., \& Lettenmaier, D. P. (2012). Relationship between hourly extreme precipitation and local air temperature in the United States. Geophys. Res. Lett. , 39, L16403.

Molnar, P., Fatichi, S., Gaal, L., Szolgay, J., \& Burlando, P. (2015). Storm type effects on super Clausius-Clapeyron scaling of intense rainstorm properties with air temperature. Hydrol. Earth Syst. Sci. , 19, 1753-1766.

O'Gorman, P. A., \& Muller, C. G. (2010). How closely do changes in surface and column water vapour follow Clausius-Clapeyron scaling in climate change simulations? Enviromental Reserch Letters , 5, 025207.

Pall, P., Allen, M. R., \& Stone, D. A. (2007). Testing the Clausius-Clapeyron constraint on changes in extreme precipitation under CO2 warming. Clim. Dyn. , 28, 351-363.

Panthou, G., Mailhot, A., Laurence, E., \& Talbot, G. (2014). Relationship between surface temperature and extreme rainfalls: a multitime-scale and event-based analysis. J.Hydrometeorol , 15, 1999-2011.

Shaw, S. B., Royem, A., \& Riha, S. J. (2011). The relationship between extreme hourly precipitation and surface temperatures in different hydroclimatic regions of the United States. $J$. Hydrometeorol , 12, 319-325.

Sherwood, S. C., Roca, R., Weckwerth, T. M., \& Andronova, N. G. (2010). Tropospheric water vapor, convection, and climate. Rev. Geophys. , 48, RG2001.

Utsumi, N., Seto, S., Kanae, S., Maeda, E., \& Oki, T. (2011). Does higher surface temperature intensify extreme precipitation. Geophys. Res. Lett. , 38, L16708.

Wentz, F. J., Ricciardulli, L., Hilburn, K., \& Mears, C. (2007). How much more rain will global warming bring? Science, 317, 233-35.

Westra, S., Fowler, H. J., Evans, J. P., Alexander, L. V., Berg, P., Johnson, F., et al. (2014). Future changes to the intensity and frequency of shortduration extreme rainfall. Rev. Geophys, 52, 522-555.

$\mathrm{Yu}, \mathrm{R} .$, \& Li, J. (2012). Hourly rainfall changes in response to surface air temperature over eastern contiguous China. J. Clim , 25, 6851-6861. 